\title{
Diagnosis and treatment of Herlyn-Werner-Wunderlich syndrome: a case report
}

\author{
Kinga Wdowiarz, Adrianna Skrajna, Małgorzata Reinholz-Jaskólska
}

Department of Obstetrics and Gynaecology, Praski Hospital, Warsaw, Poland

\begin{abstract}
Introduction: Herlyn-Werner-Wunderlich (HWW) syndrome is a rare congenital anomaly characterized by uterus didelphys with blind hemivagina and ipsilateral renal agenesis. It is one of the rare anomalies of the genitourinary system and is usually identified in the first few years after menarche.

Case report: An 18-year-old patient presented with painful menses for 1 year and was suspected for vaginal haematoma. Symptoms appeared 5 years after menarche.

Discussion: Magnetic resonance imaging of the pelvis revealed a congenital anomaly of the genital tract. Two steps of surgical management were performed. First, a hysteroscopic incision of the vaginal septa and evacuation of vaginal haematoma were performed. Subsequently, the vaginal septum was partially excised during laparohysteroscopy.

Conclusions: Ipsilateral renal agenesis was confirmed by ultrasound during hospitalization, and the diagnoses of obstructed hemivagina and ipsilateral renal anomaly syndrome were established.

The aim of this study was to present the diagnostic and therapeutic approaches in HWW syndrome, and to draw attention to the challenges in its diagnosis, which can coexist with multiple complications.
\end{abstract}

Key words: Herlyn-Werner-Wunderlich syndrome, genitourinary system, hemivagina, ipsilateral renal anomaly.

\section{Introduction}

Obstructed hemivagina and ipsilateral renal anomaly (OHVIRA), also known as Herlyn-Werner-Wunderlich (HWW) syndrome, is a very rare congenital anomaly of the genitourinary system. It is characterized by the presence of uterus didelphys with blind hemivagina and ipsilateral renal agenesis [1, 2] (Fig. 1). The aetiology is associated with the improper development of the Mullerian and Wolff ducts [3]. The first symptoms usually present after menarche and are nonspecific, leading to delayed diagnosis. The most common clinical features include dysmenorrhoea, recurrent lower abdominal pain, and haematocolpos [4, 5]. Diagnosis of OHVIRA syndrome is based on radiological examination combining ultrasound and magnetic resonance imaging (MRI) [1]. Earlier diagnosis and plastic surgical management of the vaginal septum decrease the pain and prevent complications $[4,6]$.

\section{Case report}

An 18-year-old woman was admitted to our hospital for further management of a genital tract anomaly. Two months earlier, an MRI had been performed for dysmenorrhoea lasting for a year. The MRI revealed 2 separated uterine bodies and cervices. The anatomy of the vagina was difficult to view. Left-sided haematocolpos/ haematometrocolpos was noted $(66 \times 43 \times 67 \mathrm{~mm})$. Both ovaries were noted to have small follicles; the dimensions of the right ovary were $31 \times 18 \times 25 \mathrm{~mm}$, and those of the left ovary were $20 \times 34 \times 21 \mathrm{~mm}$ (Figs. 2, 3).

The patient attained menarche at 12 years of age. After menarche the patient experienced slightly heavy
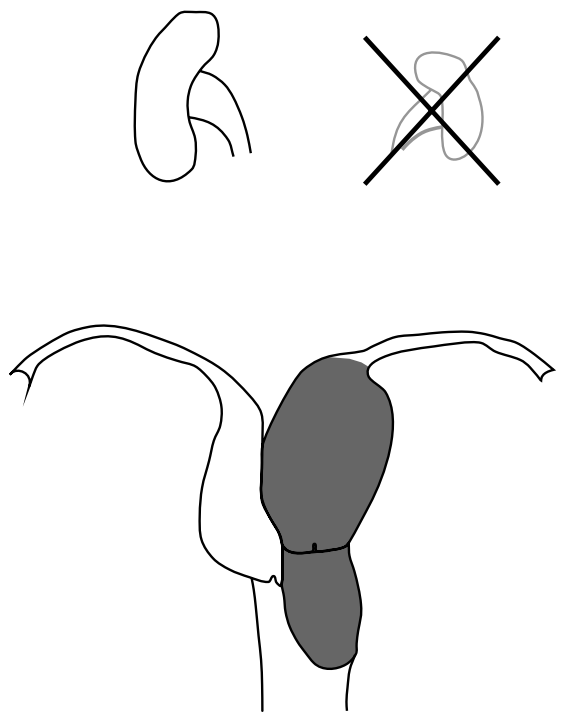

Fig. 1. A graphic representation of OHVIRA syndrome 


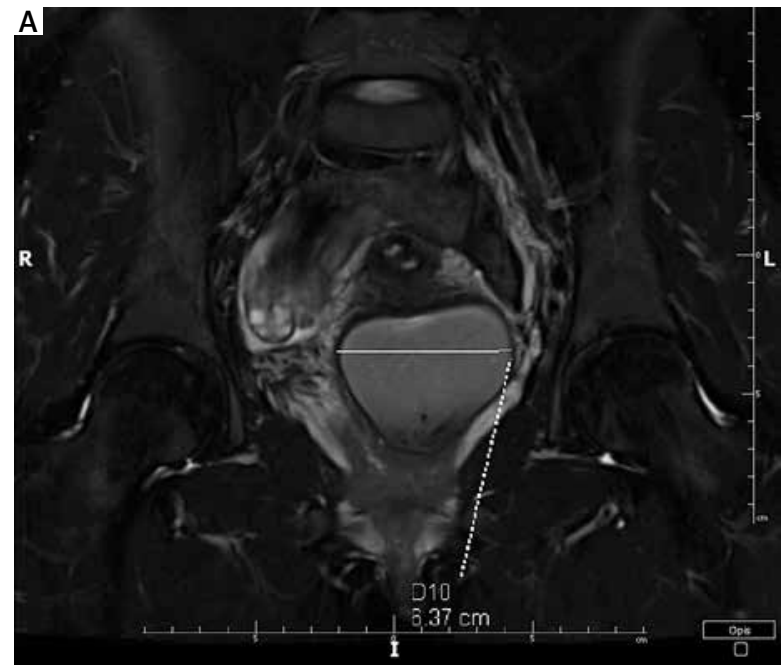

Fig. 2. MRI findings in our patient with OHVIRA syndrome

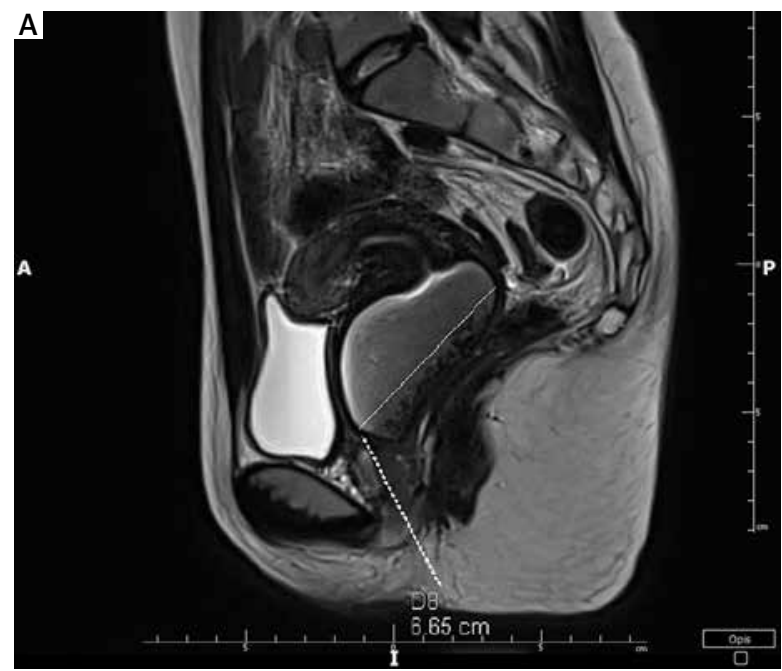

Fig. 3. MRI findings in our patient with OHVIRA syndrome

and painful regular menstruation every 28 days that lasted for 7 days each.

Menstrual history the year before the investigations revealed successively increased pain with each cycle, reaching a peak at the $3^{\text {rd }}$ or $4^{\text {th }}$ cycle, during which she experienced very heavy and painful menstruation. A brief spontaneous relief of symptoms was followed by successively increased pain in the manner described before. The patient was not sexually active at the time of the investigation. She denied chronic diseases, medication intake, or any surgical operations.

A 6-cm lump above the anterior vaginal wall on the left side was felt on bimanual examination. Ultrasound examination revealed the lump to be a cystic lesion with heterogenic echogenicity of the fluid content (dimensions $60 \times 50 \mathrm{~mm}$ ). Diagnostic hysteroscopy under general anaesthesia was considered.

Hysteroscopic examination revealed normal vaginal walls. A small cervix was observed to the right side
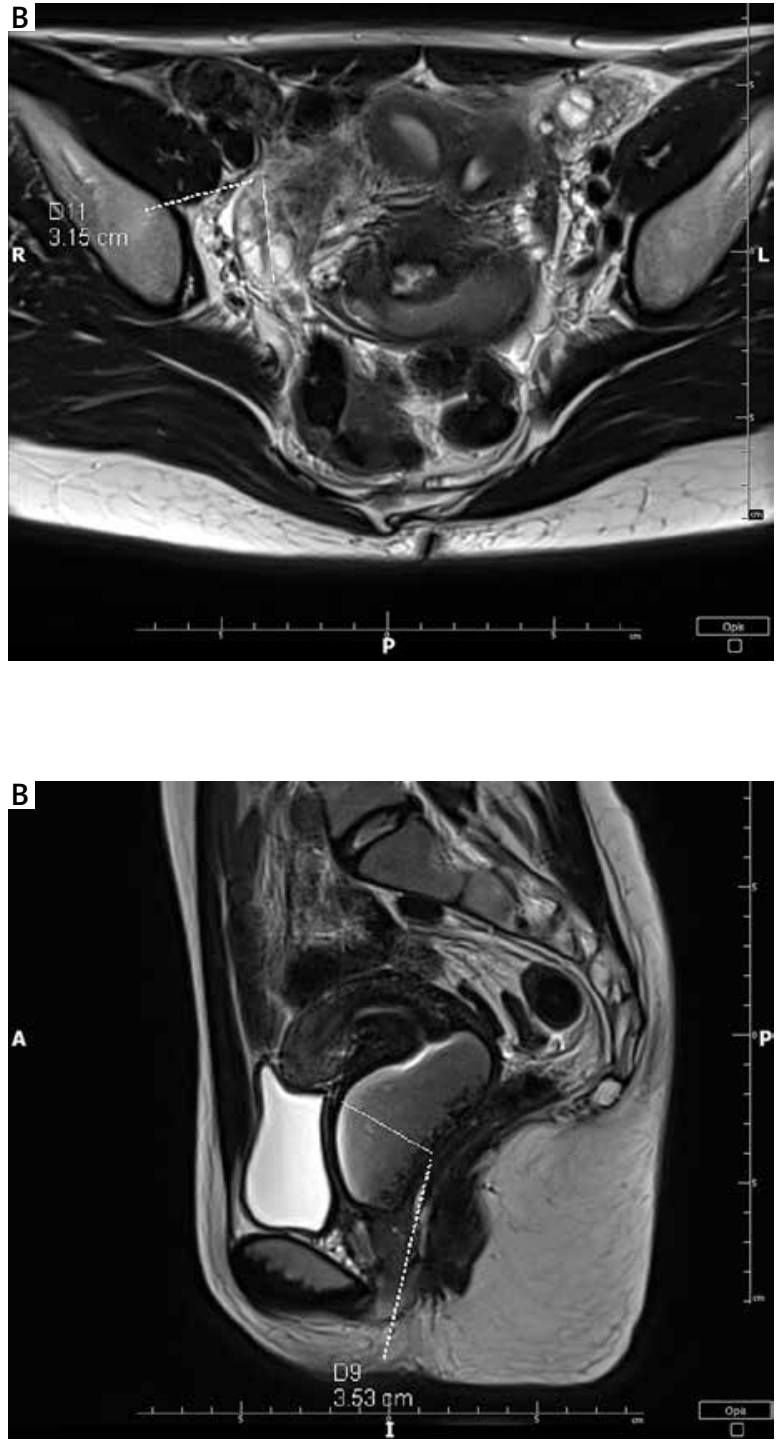

of the vaginal vault. A hysteroscope was introduced through the cervical canal and reached the uterine cavity. The uterine cavity was lined with normally shaped endometrium and uterine orifice of the Fallopian tube, shown on the right side. Ultrasound-guided aspiration of the fluid in the lump was performed. The fluid had chocolate-like colour and consistency as that of haematoma. The lump's interior was rinsed with normal saline. Ultrasound examination revealed 2 uterine cavities - the right one was filled with fluid and the left one remained empty. Both cavities had normal endometrial lining. Further extension of the surgery was halted due to bladder injury risk and performance of the procedure in the hysteroscopic unit. The patient was advised to undergo the second step of surgery in the main operating theatre.

Two months later the patient was followed up and had no complaints of painful menstruations. Ultrasound examination showed a recurrent haematoma at 


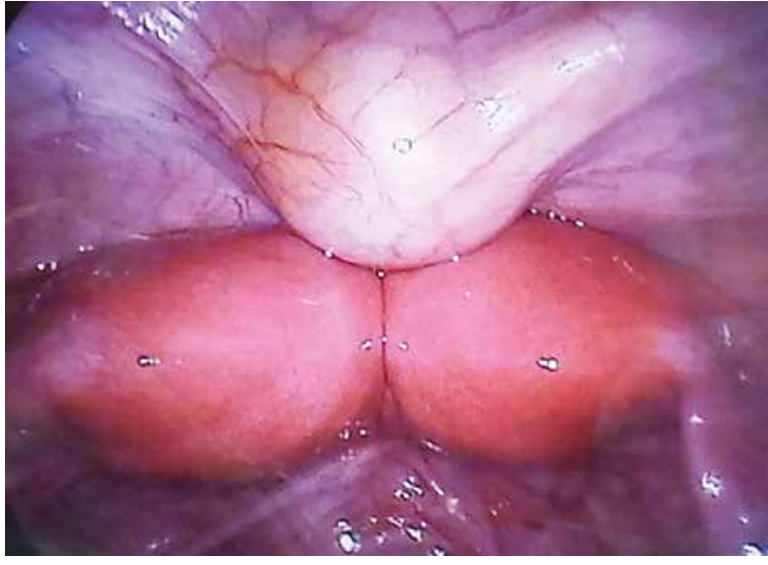

Fig. 4. Laparoscopic view of the uterus didelphys

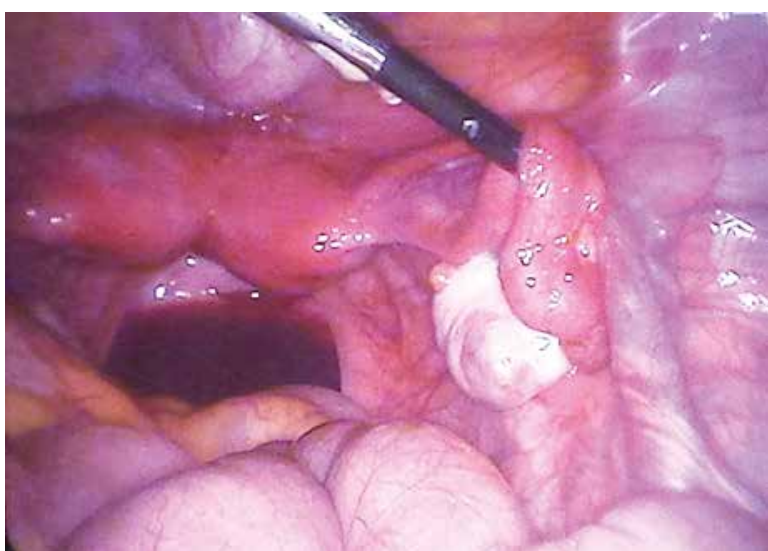

Fig. 5. Laparoscopic view of a normal right ovary

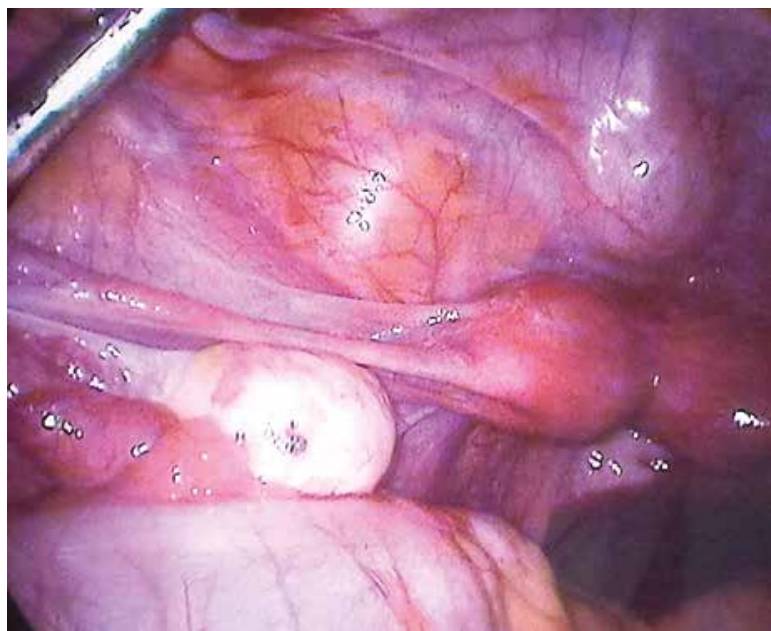

Fig. 6. Laparoscopic view of a normal left ovary

the same location. Additionally, the second step surgery was postponed due to relief of symptoms. Eight months later, incremental dysmenorrhoea was experienced, and the patient was hospitalized for further management by laparohysteroscopy.

Laparoscopic surgery confirmed 2 separated uterine bodies, which were fused at the level of internal cervical orifices. Both uterine bodies had regular outlines and were mobile. Each Fallopian tube was attached typically to its corresponding uterine body. Both adnexa were without macroscopic changes (Figs. 4-6). Then a hysteroscope was introduced through the vagina, and the right-sided component of the uterus was visualized. The total length of the right cervix and uterus was $6 \mathrm{~cm}$. The hysteroscope was introduced through the incision made for the previous procedure to a pouch filled with haematoma. The haematoma was evacuated and the hysteroscope revealed a small uterine cervix. The hysteroscope was introduced further to the left-side uterine cavity and showed the uterine orifice of the left Fallopian tube. The total length of the left-sided uterine body and left cervix was $8 \mathrm{~cm}$. Excision of the partial vaginal septum was performed. The septum was the wall of the blinded space where the haematoma collected. The edges of the wound were sutured by continuous sutures. Bilateral tubal patency was confirmed with methylene blue. The postoperative period was uncomplicated.

Abdominal ultrasound was performed to identify other possible anomalies. Renal agenesis was diagnosed on the same side of the vaginal septum. The right kidney was typically located with normal echostructure and a total length of $14 \mathrm{~cm}$. Diagnosis of OHVIRA syndrome was established depending on the characteristic congenital anomalies. At the 3-month postoperative follow-up of the patient, no abnormal mass was felt or observed by ultrasound and the patient did not complain of any recurrence of symptoms.

\section{Discussion}

OHVIRA syndrome is a very rare congenital anomaly of the genitourinary system, with an incidence of $0.1-3.8 \%$ [7]. It is characterized by uterus didelphys with blind hemivagina and ipsilateral renal agenesis [1, 2]. Other congenital anomalies may occur, and these include dysplastic or atrophic kidney and ectopic ureter with insertion to blinded hemivagina [8]. OHVIRA syndrome is considered as the most common obstructive uterine anomaly [9]. The exact pathogenesis is not fully understood. It is associated with improper fusion of the Mullerian ducts during the $8^{\text {th }}$ week of gestation and improper development of the Wolf ducts [3]. There are also hypotheses regarding a potential common origin of Mullerian anomalies and endometriosis; the main 2 hypotheses are the Mullerian rests hypothesis and that of coelomic metaplasia. It proposes that the presence of cells of Mullerian origin within the peritoneal cavity could be induced to form endometrial tissue. Some undetermined stimulus, either hormonal, infectious, or environmental, is believed to provoke metaplastic changes in the peritoneal lining, resulting in endometrial implants [10]. A case of uterine didelphys 
with vaginal septum was first described in 1922 [11] In the 1970s, Herlyn, Werner, and Wunderlich described such a uterine anomaly associated with renal agenesis. Since 2007 the triad has been called OHVIRA syndrome [12].

In 2015, Zhu et al. proposed a detailed classification depending on the analysis of patients with HWW syndrome in Peking Union Medical College Hospital [13]. The classification depends on the presence of complete or incomplete septum between vaginas and on the presence or absence of communication between uterine cavities. Four types were identified according to the proposed classification. In type 1.1 there is no communication between the uterine cavities or vaginas. Symptoms among such patients usually start few months after menarche. In type 1.2 the closed cervix is embedded in a blindly ended vagina. Symptoms begin similar to the previous group. In type 2.1 there is communication between both vaginas; therefore, the pain usually starts a few years after menarche. Additionally, genital tract infections are a complaint in such cases. In type 2.2 the vaginal septum is complete, but there is communication between the cervices or blindly ended vagina and the second cervix. Our patient mostly classified as type 2.2. The first symptoms appeared 5 years after menarche. The pain successively increased for a few cycles until the haematoma was evacuated spontaneously under the effect of pressure and volume, resulting in heavy menstruation and a decrease of pain. No blood leak was confirmed across the vaginal septum.

A literature review conducted by Milan University showed a two-fold prevalence of right-side anomaly (91 out of 138 cases) [14]. In our case, the anomaly was confirmed on the left side. According to the literature, the first symptoms can present between 5 months and 34 years of age, most commonly within a few months of menarche [5, 15]. Reports of girls aged between 5 months and 4 years having urine retention or masses in the lower abdomen that required laparoscopic treatment are present [15]. Additionally, prenatal diagnosis of the absence of one kidney followed by postnatal confirmation of genital anomalies have been reported [15].

In India, a 34-year-old woman was reported to have OHVIRA syndrome during investigations for infertility. Furthermore, endometrioid left ovarian cyst was diagnosed, as well as the characteristic triad of the syndrome [5].

However, most cases are diagnosed at between 13 and 25 years of age [13]. The most common symptoms and signs of OHVIRA syndrome are lower abdominal pain, dysmenorrhoea, and tender vaginal mass [4]; our patient had all of these. Additionally, patients with this syndrome may have recurrent urinary tract infections, urine retention, or chronic pelvic pain [16]. Nontypical clinical features might be the leading symptoms and signs in patients with OHVIRA syndrome. Zivkovic et al. described a case of a 30-year-old woman being investigated for infertility [12]. In this case, right renal agenesis had been diagnosed in her childhood. MRI confirmed uterine septum with no additional abnormalities. Thereafter, the septum was excised by laparohysteroscopy; subsequently, the patient was pregnant, and only after labour was vaginal septum confirmed, and the whole case was regarded as a rare subtype of OHVIRA syndrome.

Early diagnosis and fast surgical intervention prevent complications. MRI and ultrasound are the gold standard in the diagnosis of OHVIRA syndrome. Laparoscopy can be an additional diagnostic method when the MRI results are not clear [1, 2]. Surgical treatments depend on excision of the vaginal septum or its incision and marsupialization, which ensures outflow of the menstrual blood and considerably decreases the pain associated with haematocolpos [4, 6]. Remote complications of delayed treatment include endometriosis, adhesions in the minor pelvis, infertility, pyosalpinx, haematomas, and abscess of vagina and/or uterus [15].

Fertility of patients with OHVIRA syndrome with early diagnosis and treatment is usually not impaired [17]. Some authors mentioned that in $87 \%$ of HWW syndrome cases pregnancy could be achieved and $62 \%$ of them had delivery at term [16]. However, premature labour in patients with OHVIRA syndrome occurs more commonly compared than in the general population, although the prevalence of miscarriages does not differ [16]. Patients with OHVIRA syndrome can deliver vaginally. However, approximately $84 \%$ of pregnancies require caesarean section. The most common indication is breech presentation, which is reported in $51 \%$ of cases [16]. Foetal growth restriction is a possible complication that can increase the rate of caesarean section [16].

\section{Conclusions}

In summary, diagnosis of OHVIRA syndrome diagnosis can be a challenge for gynaecologists. Gynaecologists, paediatricians, and radiologists should consider OHVIRA syndrome and its differential diagnosis in genital tract anomalies.

\section{Disclosure}

The authors report no conflict of interest.

\section{References}

1. Orazi C, Lucchetti MC, Schingo PM, et al. Herlyn-Werner-Wunderlich syndrome: uterus didelphys, blind hemivagina and ipsilateral renal agenesis. Sonographic and MR findings in 11 cases. Pediatr Radiol 2007; 37 (Suppl. 7): 657-665.

2. Candiani GB, Fedele L, Candiani M. Double uterus, blind hemivagina, and ipsilateral renal agenesis: 36 cases and long-term follow-up. Obstet Gynecol 1997; 90 (Suppl. 1): 26-32. 
3. Rechberger T, Kulik-Rechberger B. Congenital anomalies of the female reproductive tract - diagnosis and management. Ginekol Pol 2011; 82: 137-145.

4. Smith NA, Laufer MR. Obstructed hemivagina and ipsilateral renal anomaly (OHVIRA) syndrome: management and follow-up. Fertil Steril 2007; 87 (Suppl. 4): 918-922.

5. Aswani $Y$, Varma R, Choudhary P, et al. Wolffian origin of vagina unfolds the embryopathogenesis of OHVIRA (Obstructed Hemivagina and Ipsilateral Renal Anomaly) syndrome and places OHVIRA as a female counterpart of Zinner syndrome in males. Pol J Radiol 2016; 81: 549-556.

6. Gholoum S, Puligandla PS, Hui T, et al. Management and outcome of patients with combined vaginal septum, bifid uterus, and ipsilateral renal agenesis (Herlyn-Werner-Wunderlich syndrome). J Pediatr Surg 2006; 41 (Suppl. 5): 987-992.

7. Burgis J. Obstructive Müllerian anomalies: case report, diagnosis, and management. Am J Obstet Gynecol 2001; 185 (Suppl. 2): 338-344.

8. Schlomer B, Rodriguez E, Baskin L. Obstructed hemivagina and ipsilateral renal agenesis (OHVIRA) syndrome should be redefined as ipsilateral renal anomalies: cases of symptomatic atrophic and dysplastic kidney with ectopic ureter to obstructed hemivagina. J Pediatr Urol 2015; 11: 77.e1-6.

9. Kapczuk K, Friebe Z, Iwaniec K, et al. Obstructive Müllerian anomalies in menstruating adolescent girls: a report of 22 cases. J Pediatr Adolesc Gynecol 2018; 31 (Suppl. 3): 252-257.

10. Lagana A, Garzon S, Götte M, et al. The pathogenesis of endometriosis: molecular and cell biology insights. J Mol Sci 2019; 20: 5615

11. Buttram VC Jr, Gibbons WE. Müllerian anomalies: a proposed classification (an analysis of 144 cases). Fertil Steril 1979; 32 (Suppl. 1): 40-46.

12. Živkovic K, Prka M, Živkovic N, et al. Unusual case of OHVIRA syndrome with a single uterus, unrecognized before labor and followed by an intrapartal rupture of obstructed hemivagina. Arch Gynecol Obstet 2014; 290 (Suppl. 5): 855-858.

13. Zhu L, Chen N, Tong JL, et al. New classification of Herlyn-Werner-Wunderlich syndrome. Chin Med J (Engl) 2015; 128 (Suppl. 2): 222-225.

14. Vercellini P, Daguati R, Somigliana E, et al. Asymmetric lateral distribution of obstructed hemivagina and renal agenesis in women with uterus didelphys: institutional case series and a systematic literature review. Fertil Steril 2007; 87 (Suppl. 4): 719-724.

15. Angotti R, Molinaro F, Bulotta AL, et al. Herlyn-Werner-Wunderlich syndrome: an "early" onset case report and review of literature. Int J Surg Case Rep 2015; 11: 59-63.

16. Cappello S, Piccolo E, Cucinelli F, et al. Successful preterm pregnancy in a rare variation of Herlyn-Werner-Wunderlich syndrome: a case report. BMC Pregnancy Childbirth 2018; 18 (Suppl. 1): 498.

17. Heinonen PK. Clinical implications of the didelphic uterus: long-term follow-up of 49 cases. Eur J Obstet Gynecol Reprod Biol 2000; 91 (Suppl. 2): 183-190. 